\title{
Numerical study of Lancet Furrows Dynamics Behaviour in Greater Horseshoe Bats
}

\author{
Weikai $\mathrm{He}^{1}$, Jianxiong Feng ${ }^{2} \dagger, \mathrm{Li} \mathrm{Gao}^{1}$ \\ 1) School of Physics, University of Jinan, Jinan 250000, Shandong,
}

China

2) Department of Mechanical Engineering, Virginia Tech, Blacksburg, VA

$$
\text { 24061, USA }
$$

The greater horseshoe bat (Rhinolophus ferrumequinum) emits biosonar pulses nasally. The nostril of bats has complicated noseleaf structures, which consists of lancet, anterior leaf, sella. For the three noseleaf parts, lancet is the only one that stays away from the nostril. In addition, lancet has several furrows on the surface. It is known that the furrows in static lancet possess frequency selection characteristics, however, the effects of furrows during the lancet deformation have yet to be investigated. In this study, the 3D noseleaf models were obtained by the 3D scanning of the greater horseshoe bats noseleaf sample. Then, finite element method (FEM) was used to simulate the biosonar beampattern under different degrees of lancet flexion with both natural furrow condition and voxel-filled furrow. From the numerical results, it was found that the lancet furrow can enhance the mainlobe energy gain, and regulate the sidelobes position in the acoustic beam. The results indicate that the lancet furrows in greater horseshoe bats can be critical in targeting the objectives as well as gathering environmental information around the target.

Key words: greater horseshoe bats, lancet furrows, lancet deformation, biosonar beam patterns.

Funding: This work was supported by National Science Foundation of China (11404140; 11374193; 451069; 61271453), Shandong Province (BS2013SF001) and University of Jinan (XBS1332; XBS160).

$\dagger$ Corresponding author. E-mail: jxfeng2016@gmail.edu; Phone number: +1 5407503890 


\section{Introduction}

Most species of bats have developed the active and passive biosonar system to adapt to their living environment [1,2], in which the active sonar is the sonar pulses emitted by bats, while the passive sonar is the environmental acoustic signals. In the anatomical point of view, after long-term evolution, some species of bats have developed extremely sophisticated acoustic signal emitting organs, especially for the bats that emit sonar pulses nasally $[3,4,5]$, in which the greater horseshoe bat is the species that falls into the category [6]. The greater horseshoe bats possess typical noseleaf structures, which contain lancet, anterior leaf, and sella. The sella and anterior leaf locate near the nostril, while the lancet locates away from the nostril. The lancet has delicate furrow structures on it. It has been shown that the lancet furrow is a resonance cavity that have one open end, and it shows frequency-selective characteristics [4]. The resonance cavity also shape the sonar beam in certain direction [4].

The echolocation system of bats mainly consists of emitting and receiving parts as well as nerve system [7]. The receiving parts (outer ear) is critical in the transmission of the reflected wave [8]. The surrounding substructures of the emitting parts play an important role in determining the biosonar beam pattern [9, 10]. For example, after the nose of Rhinolophus paradoxolophus bat was shortened, the focusing effect of it on the sonar beam worsened a lot, however, man-made extension can enhance the focusing effect to a little extent [9]. Dieter Vanderelst studied the side lobe changed in beampattern of $\mathrm{M}$. microtis bats and $\mathrm{P}$. discolor bats with lancet motion [10].

The previous study showed that the lancet can undergo non-rigid deformation [11]. The deformation of the lancet can enlarge the sensing area in elevation by generating obvious sidelobes in the biosonar beam, which will facilitate bats to detect the surrounding information around the main target [11]. The lancet has one pair of parallel transverse furrows on each side $[12,13]$, which is an important feature for bats categorization. The previous study shows that the static lancet furrows can change the width of the emitted ultrasonic beam [4, 12]. However, the effects of lancet in the dynamic process on the biosonar beam pattern, which is the main research objective in the current study, has yet to be explored.

The current study performed numerical investigation on the acoustic characteristics of the lancet furrows in greater horseshoe bats. The results in this study revealed the physical underpins of the lancet furrow effects on beam patterns in lancet dynamics, thus providing the fundamental data for later research on the biological properties of the lancet furrow system as well as bio-inspired application of the physics.

\section{Experiment and Methods}

Two bats, one male, one female, were used for the experiment in this study 
(captured in Lantai Mountain, Jinan, Shandong, China). To obtain the effect of the lancet furrows on the biosonar beampattern in lancet dynamics, one of the feasible method is using microphone array to measure the ultrasonic pulses. However, on one hand, the head rotation of bats will affect the accuracy of the measurement. On another hand, with filled paraffin wax in the nostril, the behavior of the bats will be affected, thus making the fidelity of the experimental data less believable. In this case, the numerical simulation method was adopted in this study instead of experimental method. Micro-CT (Skyscan 1072; Bruker microCT, Kontich, Belgium) was used to scan the bat noseleaf, thus reconstructing a 3D model for FEM simulation [11]. The individual 3D digital noseleaf models were handled using the software 3DSMax (Autodesk, Mill Valley, CA, USA), to provide a good fit to the subsequent frame positions of the bend lancet from video recordings of lancet rotation (Fig. 1). Then, numerical method (finite element method) was utilized to simulate the radiation pattern in different cases [5].

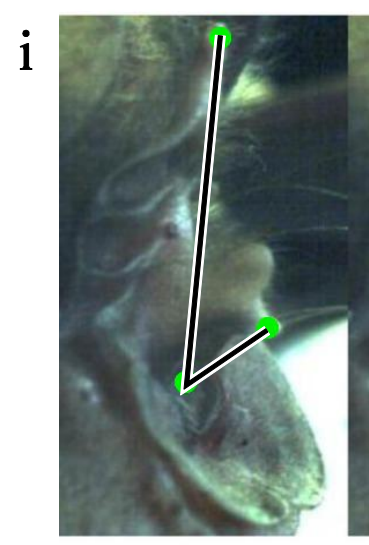

a

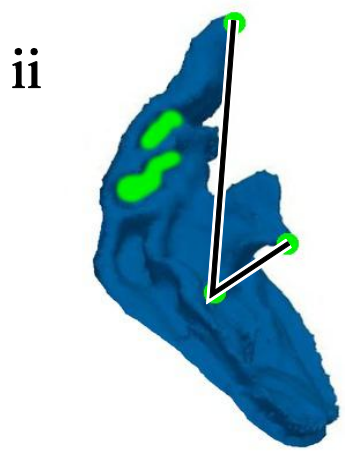

$a^{\prime}$

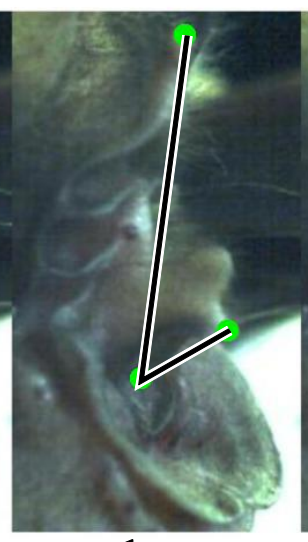

b

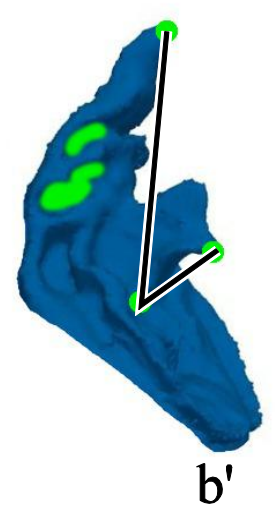

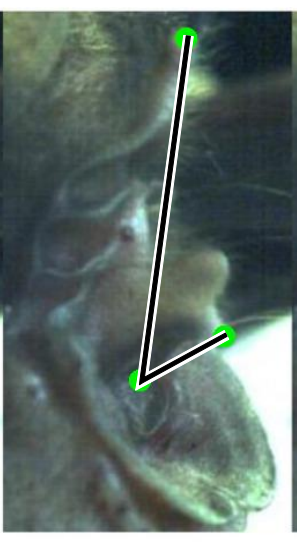

C

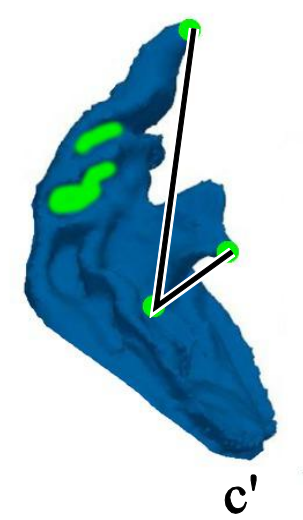

a b c d

$\uparrow \uparrow 1$

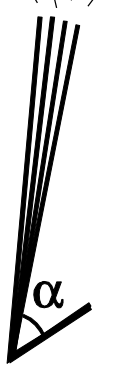

d

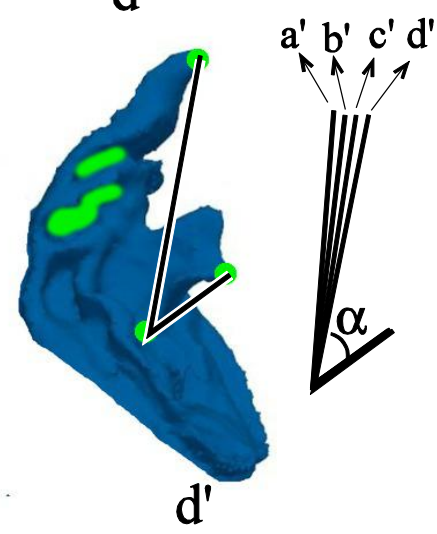

Fig. 1 The lancet deformation pattern. The four sequential frames from the initial condition to maximum deformation. The lancet furrows in each frame are filled with voxels. " $i$ " is the image of read lancet, "ii" is the $3 \mathrm{D}$ reconstructed lancet. a: $0^{\circ}$ flexion, b: $0^{\circ}$ flexion, c: $8^{\circ}$ flexion, d: $12^{\circ}$ flexion.

To analyze the acoustic effect of transverse furrows in lancet deformation quantitatively on the ultrasonic radiation, the overlap rate of acoustic lobes between adjacent frequencies is chosen for the analysis. The overlap rate is defined as the lobes 
overlap area between the neighboring frequencies over the total area of acoustic beam [7].

\section{Results}

The simulation results from natural (Fig. 2a, Fig. 3a) and filled furrows (Fig. 2b, Fig. 3b) in lancet differ significantly. To make the difference easier to be compared, in Fig. 4, the $-3 \mathrm{~dB}$ contour lines in different furrow conditions are put together in one graph. It is evident that in the low frequency range $(60-65 \mathrm{kHz})$, when the lancet flexion goes from $0^{\circ}$ to $4^{\circ}$, there is not much difference in the mainlobe area between the filled furrow and natural furrow. However, with the increase in the flexion of the lancet $\left(8^{\circ}\right.$ and $\left.12^{\circ}\right)$, the area of mainlobe with the furrow filled becomes much smaller than that of the natural furrow. It can also be observed from Fig. 4 that with the increase of the frequency $(70 \mathrm{kHz}, 75 \mathrm{kHz}, 80 \mathrm{kHz})$, the mainlobe area for the filled furrow shrink much faster than that of the natural furrow (FIg. 4), which means that the furrows decrease the lobe sensitivity to the frequency change.
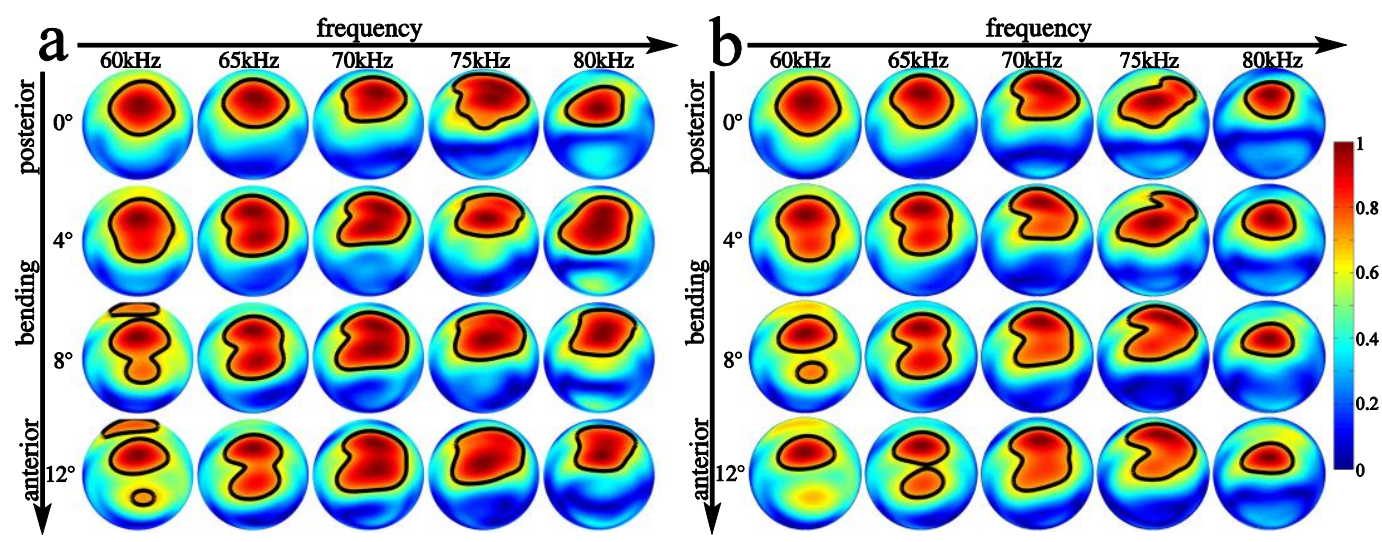

Fig .2 Numerical beampatterns obtained for lancet rotation - model 1. (a) Natural furrow condition; (b) filled furrow condition. Each row corresponds to different lancet flexion, each column corresponds to different frequencies.
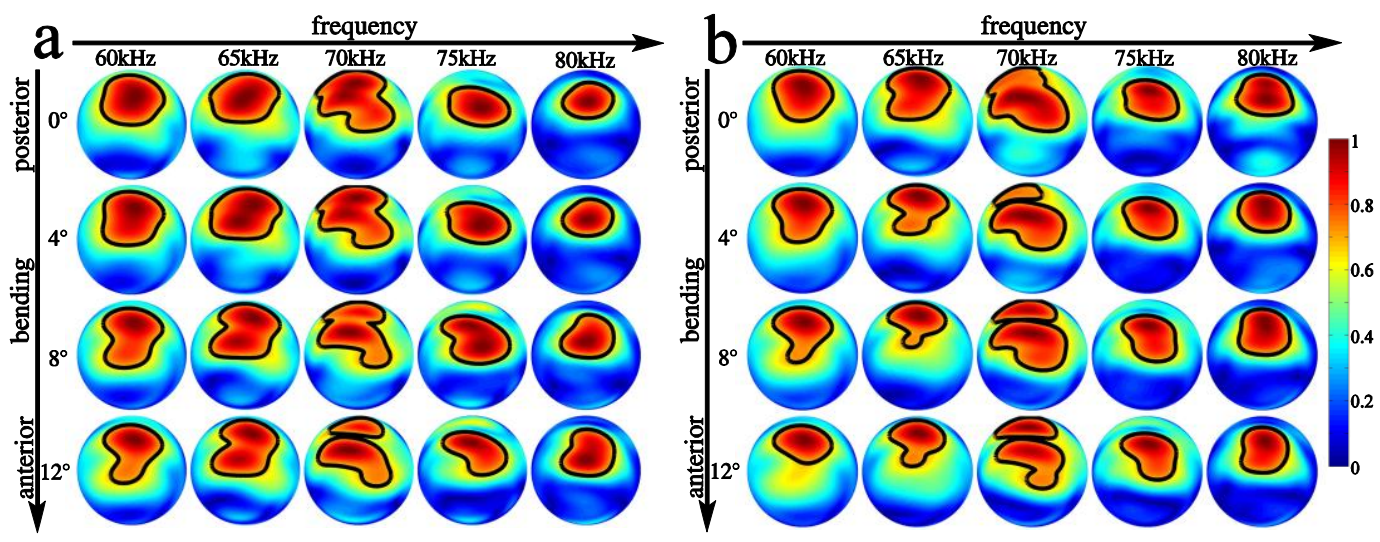

Fig .3 Numerical beampatterns obtained for lancet rotation - model 2. (a) Natural furrow condition; (b) filled furrow condition. Each row corresponds to different lancet flexion, each column corresponds to different frequencies. 


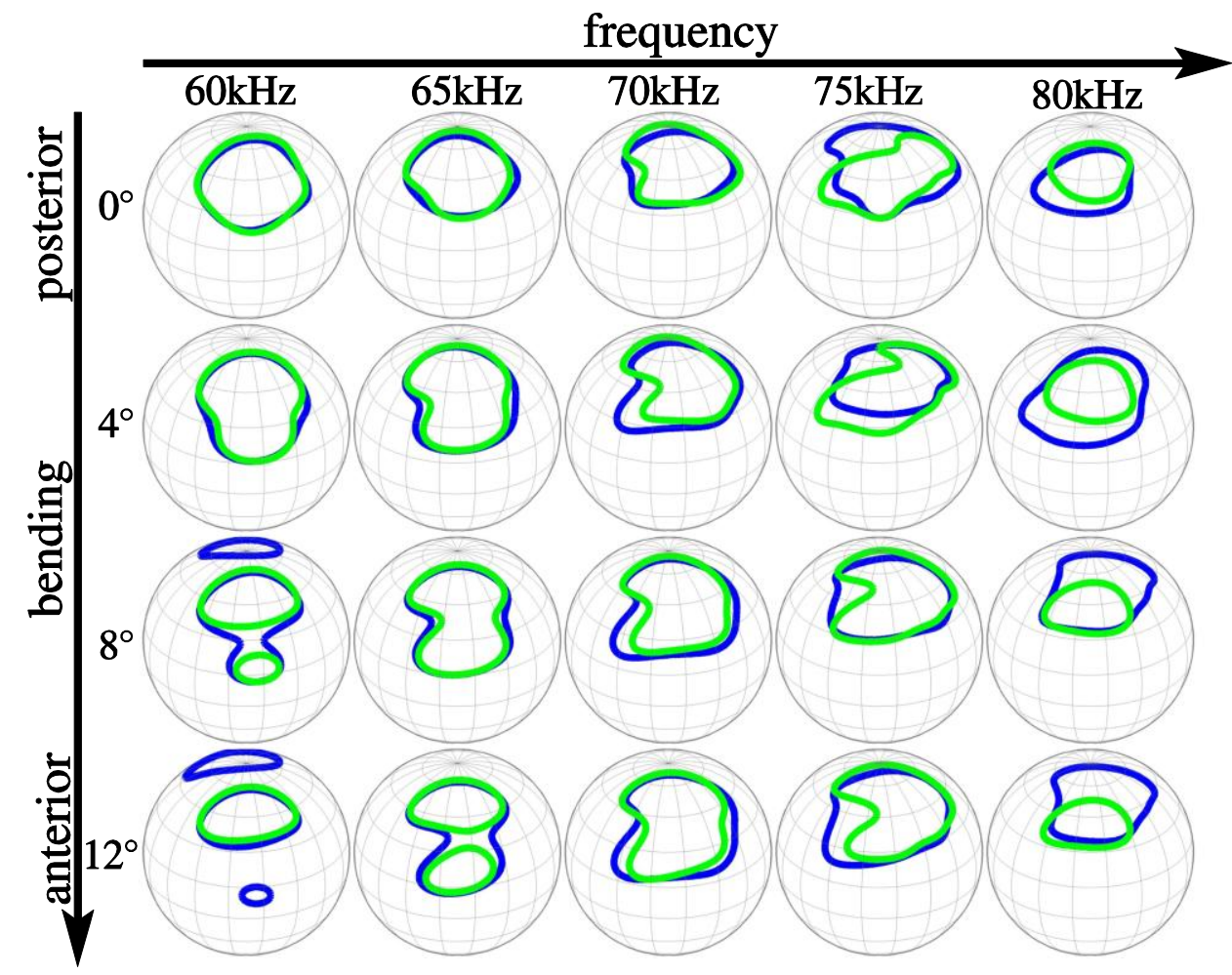

Fig. 4 Numerical estimated beampattern -3dB contour lines - model 1. The contours of both natural furrow (blue lines) and filled furrows (green lines) are put together for comparison.

For the natural furrow in the static lancet, there is a conspicuous mainlobe and a faint sidelobe in the biosonar beam. Then, with the increase in the lancet flexion, the sidelobe energy increases obviously. In Fig. 2a and Fig. 3a, it can be found that the position change of sidelobe is not regular as mainlobe. For the filled lancet furrow, conspicuous sidelobes appear with the increase in the lancet deformation in each frequency. To investigate the effects of furrows on the biosonar beam detailedly in lancet dynamics, the overlap rates of the mainlobes and the maximum gain sidelobes between the adjacent frequencies were calculated (Fig. 5). The overlap rate is a criterion of measuring the relative change of the biosonar beam, because the change of the biosonar beam is negatively correlated with the overlap rate. For $80 \%$ of the frequencies, the beampattern in the natural furrow condition shows an overlap rate that is higher than $40 \%$ (Fig. 5a). However, after the furrow is filled, only $65 \%$ of the frequencies has the overlap rate that is higher than $40 \%$. 

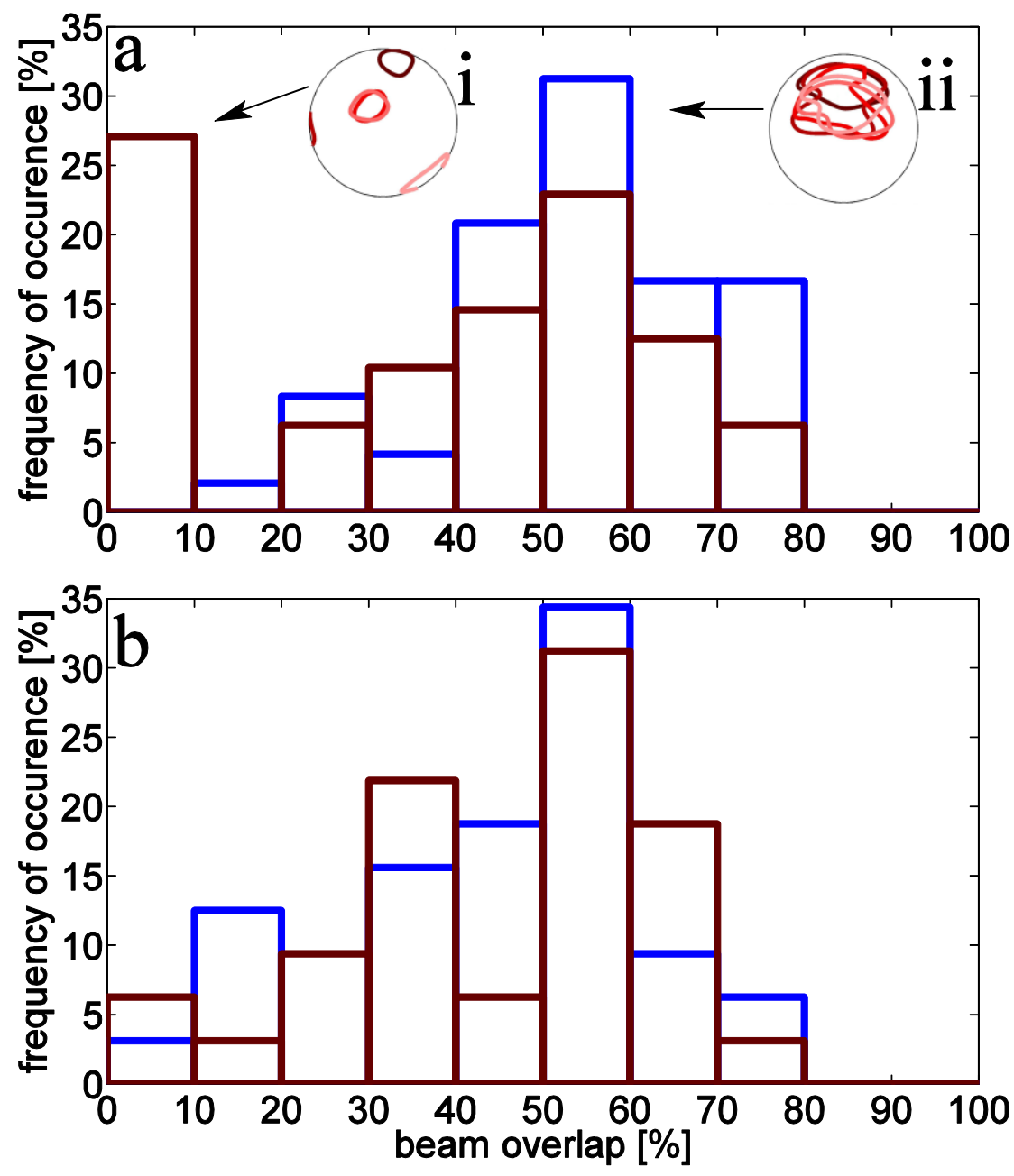

Fig 5 Overlap rate of the mainlobe and sidelobes between different frequencies. The $\mathrm{X}$ axis represent beam overlap rate, the $\mathrm{Y}$ axis represents the occurrence frequency. The red rectangles represents sidelobes, while the blue rectangles represent the mainlobes. (a) Natural furrow condition; (b ) filled furrow condition. Letter " $\mathrm{i}$ " is the side lobe with overlap rate between $0-10 \%$, letter ii“" is the mainlobe with overlap rate between $50-60 \%$.

In Fig. 5a, it can be found out that the sidelobe overlap rates are much lower than mainlobes. More than $50 \%$ of the sidelobes have the overlap rates that are lower than $20 \%$. From Fig. 4, it can be found that with the motion of the lancet, the conspicuous sidelobes become more scattered and distribute around the mainlobes, which means that the sidelobe energy distributes in more areas with lancet motion. The scatter of the energy will facilitate the detection of the environmental information for bats. In Fig. 5b, it can be observed that, for the filled furrow, the overlap rates of the sidelobes are still lower than that of the mainlobes. However, the difference becomes much smaller compared the natural furrow case. Only $10 \%$ sidelobes have the overlap rate under $20 \%$. For the furrow filled condition, comparing the beampatterns (Fig. 2b, Fig $3 b)$ with different lancet deformation, it can be observed that, with the lancet flexion increasing, sidelobes become more obvious as the natural furrow case. However, the energy distribution of the sonar beam is not as scattered as the condition without the 
furrow filled. The mainlobe shows much less overlap after the furrow was filled, while the sidelobes does not show much change with frequency increase. This indicates that the block of the furrows will affect the ability of bats to detect the main target as well as the environmental information.

\section{Conclusion}

The previous results showed that the mainlobe in the acoustic beam can be utilized to detect the faint signal as well as identify the signal target [14]. However, the trajectory of the bat flight is not fixed in the preying activities. In the complex environments, the bat needs to adjust the outgoing acoustic signal constantly in a wide frequency and spatial direction range [15]. By the qualitative analysis of Fig. 2, Fig.3 and quantitative analysis of Fig. 5, it can be concluded that the fill of the furrows with voxel can decrease the overlap of the mainlobe and increase the overlap of sidelobe. The decrease in the mainlobe overlap will lower the sensitivity of the sensing of the acoustic signal. Besides, the overlap rate of the sidelobes increase due to the fill of the furrow, which means the change of the sidelobes area becomes smaller and the sidelobe position stays close regardless of the frequency change. The concentration of the acoustic energy is not a favorable characteristic in the detection of the environmental information. The following conclusions can be drawn from the analysis of the results: 1) the lancet furrow plays an important part in the widening of the mainlobe, thus facilitating the detection of small target; 2) the lancet furrows can enhance the complexity of the change of sidelobes with frequency change, which enables the greater horseshoe bats to detect the environmental information besides the target. This effect optimizes the utilization of the acoustic energy.

The findings in this research can be applied to the ultrasonic sensing. Electromagnetic wave is the most commonly used tool for the radar detection, robotic control etc. However, the limitation of the electromagnetic wave is driving the revolution of the basic principles of the detection theory. Currently, the electromagnetic signals used by microphones and radars are isotropic, which is not enough to provide the selectivity that can satisfy the sensing need. In this context, the ultrasonic sensing mechanisms used by bats can be an inspiration to the design of the next generation sensing devices, such as radar etc. The mechanisms of how the lancet furrows shape the ultrasonic signals can be used for the design of dynamic sensing device, thus achieving the better manipulation on the energy distribution of the outgoing signals.

\section{References}

[1] Griffin. D. R. (1986). Listening in the Dark. Ithaca: Cornell University Press. $57-147$.

[2] Neuweiler G, Metzner W, Heilmann U, Rübsamen R, Eckrich M. (1987). Foraging Behaviour and Echolocation in the Rufous Horseshoe Bat (Rhinolophus rouxi) of Sri 
Lanka. Behav Ecol Sociobiol. 20, 53-67.

[3] Griffin D. R. (1986). Listening in the Dark. Cornell University Press, Ithaca.

[4] He W, Petterson S. C, Gupta A. K, Simmons J. A, Müller R. (2015). Lancet Dynamics in Greater Horseshoe Bats, Rhinolophus ferrumequinum. PLOS ONE. 10, e0121700.

[5] Zhuang Q, Müller R. (2007), Numerical Study of the Effect of the Noseleaf on Biosonar Beamforming in a Borseshoe Bat. Phys Rev E. 76, 051902-11.

[6] Jones G, Rayner J. (1989). Foraging Behavior and Echolocation of Wild Horseshoe Bats Rhinolophus ferrumequinum and R. hipposideros (Chiroptera, Rhinolophidae). Behav Ecol Sociobiol. 25, 183-91.

[7] Jone G., Teeling E. C. (2006). The evolution of echolocation in bats. Trends Ecol. Evol. 21(3): 149-156.

[8] Müller R, Lu H, and John R. Buck. (2006). Sound-diffracting flap in the ear of a bat generates spatial information. Phys. Rev, Lett. 100(10):108701

[9] Zhang Z, Nguyen ST, Müller R. (2009). Acoustic effects accurately predict an extreme case of biological morphology. Phys Rev Lett. 103: 038701.

[10] Dieter Vanderelst, Fons De Mey, Herbert Peremans, Inga Geipel, Elisabeth Kalko, Uwe Firzlaff (2010). What Noseleaves Do for FM Bats Depends on Their Degree of Sensorial Specialization. PLoSONE. 5(8): e11893. doi:10.1371/journal.pone.0011893.

[11] He W, Petterson S. C, Gupta A. K, Simmons J. A, Müller R. (2015). Lancet Dynamics in Greater Horseshoe Bats, Rhinolophus ferrumequinum. PLOS ONE. 10 (4): e0121700.

[12] Zhuang Q, Müller R. (2006). Noseleaf Furrows in a Horseshoe Bat Act as Resonance Cavities Shaping the Biosonar Beam. Phys Rev Lett. 97: 218701-4.

[13] Feng L, Gao L, Lu H, Müller R. (2012). Noseleaf Dynamics during Pulse Emission in Horseshoe Bats. PLoS ONE. 7, e34685.

[14] Rao R. K, Ben-Arie J. (1996). Optimal Head Related Transfer Functions for Hearing and Monaural Localization in Elevation: a Signal Processing Design Perspective. IEEE Trans Biomed Eng. 43: 1093-105.

[15] Gao L, Balakrishnan S, He W, Yan Z, Müller R. (2011). Ear deformations give bats a physical mechanism for fast adaptation of ultrasonic beampatterns. Phys Rev Lett 107: 214301. 